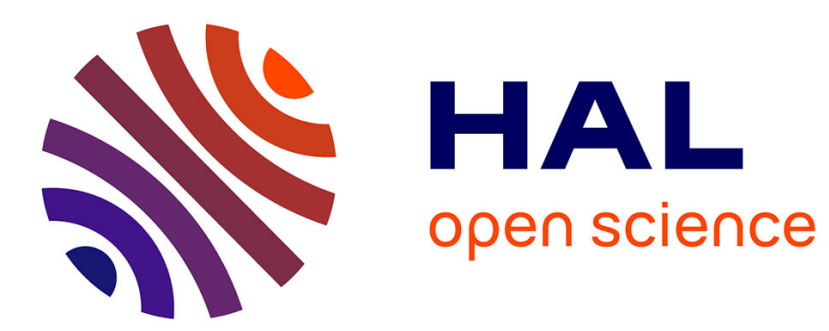

\title{
A MICROSCOPIC TRANSPORT THEORY OF ELECTRON-PHONON SYSTEMS
}

\author{
A. Wagh
}

\section{To cite this version:}

A. Wagh. A MICROSCOPIC TRANSPORT THEORY OF ELECTRON-PHONON SYSTEMS. Journal de Physique Colloques, 1981, 42 (C6), pp.C6-471-C6-474. 10.1051/jphyscol:19816137 . jpa00221201

\section{HAL Id: jpa-00221201 https://hal.science/jpa-00221201}

Submitted on 1 Jan 1981

HAL is a multi-disciplinary open access archive for the deposit and dissemination of scientific research documents, whether they are published or not. The documents may come from teaching and research institutions in France or abroad, or from public or private research centers.
L'archive ouverte pluridisciplinaire HAL, est destinée au dépôt et à la diffusion de documents scientifiques de niveau recherche, publiés ou non, émanant des établissements d'enseignement et de recherche français ou étrangers, des laboratoires publics ou privés. 
JOURNAL DE PHYSIQUE

Colloque C6, supplément au n० 12 , Tome 42 , décembre 1981

page $\quad$ 66-471

\title{
A MICROSCOPIC TRANSPORT THEORY OF ELECTRON-PHONON SYSTEMS
}

\author{
A.S. Wagh
}

Physies Department, University of West Indies, Mona, Kingston, Jamaica, W.I.

\begin{abstract}
The two-time Green functions are used to derive kinetic equations of a system of electrons and phonons interacting with Frohlich hamiltonian. A suitable connected diagram analysis is used to derive simultaneous equations similar to Kadanoff-Baym equations. The initial correlations are shown to decay in time comparable to the macroscopic time scale. The general transport equations derived in the steady state are applicable to various transport processes. It is shown that the previous assumption of considering the phonons to be in thermal equilibrium is justified only for dilute systems and phonon drag is appreciable in denser systems.
\end{abstract}

1. Introduction. The two-time Green functions have been extensively used by Kadanoff and Baym ${ }^{1}$ to study the evolution and transport of a quantum mechanical imperfect gas. We extend this formulation to the electron phonon system. A connected diagram ${ }^{2}$ analysis is used to derive the evolution equations from the theorem by Bloch and Domenicis ${ }^{3}$. We obtain four transport equations two for electrons and two for phonons, the latter describing phonon drag. Using these equations it is possible to show that the phonon drag is negligible for dilute systems such as electrons in semiconductors but can be appreciable in metals.

2. The Evolution Equations. The Frohlich hamiltonian for electron phonon interaction is given by

$$
\begin{aligned}
& H=H_{0}+\lambda V=\int\left[h_{0}(p)+\phi\left(i \frac{\partial}{\partial \underline{p}}, t\right)\right] a^{+}(\underline{p}) a(\underline{p}) d \underline{p}+\int \S p b+(\underline{p}) b(\underline{p}) d \underline{p} \\
& +\lambda \iint d \underline{p}_{1} \underline{p}_{2} \gamma_{p_{2}}^{\frac{1}{2}}\left[a^{+}\left(\underline{p_{1}}+\underline{p}_{2}\right) a\left(\underline{p}_{1}\right) b\left(\underline{p_{2}}\right)+a^{+}\left(\underline{p}_{1}\right) a\left(\underline{p}_{1}+\underline{p_{2}}\right) b^{+}\left(\underline{p_{2}}\right)\right]
\end{aligned}
$$

Here $a(\underline{p})$ and $b(\underline{p})$ stand for annihilation operators for electrons and phonons, $\phi$ is the external field and other symbols have their usual meaning. The partial Green functions $g^{2}$ for electrons and $n^{\gtrless}$ for phonons are defined by

$$
\begin{array}{ll}
g^{<}(1,2)=-i \operatorname{Tr}\left\{\rho a^{+}(2) a(1)\right\} & n^{<}(1,2)=i \operatorname{Tr}\left\{\rho b^{+}(2) b(1)\right\} \\
g^{>}(1,2)=-i \operatorname{Tr}\left\{\rho a(1) a^{+}(2)\right\} & n^{>}(1,2)=i \operatorname{Tr}\left\{\rho b(1) b^{+}(2)\right\}
\end{array}
$$


where the symbols 1 and 2 stand for $\left(\underline{p}_{1}, t_{1}\right)$ and $\left(\underline{p}_{2}, t_{2}\right)$. The evolution of $g^{\gtrless}(1,2)$ may be studied by differentiating each of these with respect to the times $t_{1}$ and $t_{2}$. One obtains for example

$$
\begin{aligned}
i \frac{\partial g^{<}}{\partial t_{1}}(1,2)= & -i \operatorname{Tr}\left\{\rho a^{+}(2) u^{+}\left(t_{1}\right)\left[a\left(\underline{p}_{1}\right), H\right] U\left(t_{1}\right)\right\} \\
= & {\left[h_{0}(1)+\mu \phi(1)\right] g^{<}(1,2)-i \operatorname{Tr}\left\{\rho \lambda \int d \underline { p } _ { 3 } \gamma _ { p _ { 3 } } \left[a^{+}\{2) a\left(\underline{p}_{1}-\underline{p}_{3}, t_{1}\right) b\left(\underline{p}_{3}, t_{1}\right)\right.\right.} \\
& \left.\left.+a^{+}(2) a\left(\underline{p}_{1}+\underline{p}_{3}, t_{1}\right) b^{+}\left(\underline{p}_{3}, t_{1}\right)\right]\right\}
\end{aligned}
$$

This equation is not in closed form. One needs to analyze it by means of perturbation and connected diagram technique, the essential features being similar to the one obtained from Wick's theorem for an imperfect gas. Here however, we start from $B$ loch and Dominicis ${ }^{3}$ theorem that the average $\langle A B C . .>$ for operators $A, B, C$, etc. may be decomposed into averages of the pair-products, viz.

$\langle\mathrm{ABC} \ldots\rangle=\sum_{\mathrm{n}}( \pm 1)^{\mathrm{n}}\langle\mathrm{AB}\rangle\langle\mathrm{CD}\rangle \ldots \ldots$

where + stands for phonons and - for electrons. The descriptions of the self-energy parts is given in the table. One obtains for $\mathrm{g}^{\gtrless}$

$$
\begin{aligned}
& i \frac{\partial g^{z}}{\partial t_{1}}=\left[h_{0}\left(p_{1}\right)+\mu \phi\left(\underline{p}_{1}\right)\right] g g^{z}+\int_{0}^{t_{2}} d t_{3} \int d p_{3} \Sigma^{z}(1,3) g \xi(3,2)-t_{1} \int^{t_{2}} d t_{3} \int d p_{3} \Sigma^{\gtrless}(1,3) g^{\gtrless}(3,2)
\end{aligned}
$$

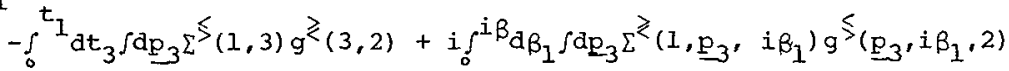

where $\Sigma^{k}=\varepsilon^{k}+\varepsilon^{ \pm}$. The last term in eq. (5) is due to initial correlations, which are due to our choice of initial time as $t_{1}=0$. In a similax manner one writes equations for $\frac{\partial \mathrm{g}^{\gtrless}}{\partial t_{2}}$ and for $\mathrm{n}^{\gtrless}$ all having same form of Kadanoff and Baym equations.

3. Decay of Initial Correlations. Though the electron-phonon interaction is a long range potential, we show here that the initial correlations die out by the time steady rate is reached.

If the system is initially in thermal equilibrium, the initial Green functions for electrons obeying classical statistics are

$g_{0}^{<}\left(\underline{p}_{1} i \beta_{1}, 2\right)=e^{-\beta_{1} \varepsilon(p)+i_{\varepsilon}(p) t_{2}+\alpha-\beta \varepsilon(p)} ; q_{0}^{>}\left(1, \underline{p}_{2}, i \beta_{1}\right)=e^{-\beta_{1} \epsilon(p)+i \varepsilon(p) t_{1}}$

where $\underline{p}=\frac{1}{2}\left(\underline{p}_{1}-\underline{p}_{2}\right), \epsilon$ and $\alpha$ are particle energy and fugacity. The initial correlation term involved in eg. (5) for a linear lattice with one atom per lattice in binary collision approximation is

$$
\begin{aligned}
& I=\int_{0}^{i \beta_{a}} d\left(i \beta_{1}\right) \int d \underline{p}_{3} \Sigma^{<}\left(1, \underline{p}_{3}, i \beta_{1}\right) g^{>}\left(-\underline{p}_{3}, i \beta_{1}, 2\right) \\
& =\lambda^{2} \int_{0}^{i \beta} d\left(i \beta_{I}\right) \int_{0}^{P m} d \underline{p^{\prime}} \gamma_{p^{\prime}} e^{\alpha-\left[\left(\beta-\beta_{1}\right) i t_{I}\right] \epsilon\left(\underline{p}-\underline{p}^{\prime}\right)} \\
& \operatorname{xexp}\left[i \S(p) t_{1}+\left(\beta-i t_{2}\right) \in(p)\right]\left\{\frac{e^{-\beta \xi\left(p^{\prime}\right)}}{e^{\beta \xi\left(p^{\prime}\right)}-1}+\frac{e^{\left(\beta+\beta_{1}\right) \S\left(p^{\prime}\right)}}{e^{\beta \xi\left(p^{\prime}\right)}-1}\right\}
\end{aligned}
$$

Here $p_{m}$ is the maximum phonon momentum obtained at the edge of the first Brillouin zone. Assuming phonon evergy to be small as compared to the electron energy and for a linear lattice 
$\S(\mathrm{p})=\S_{\mathrm{o}} \sin \frac{1}{2} \mathrm{p}^{\prime} \mathrm{a} \simeq \frac{1}{2} \S_{0} \mathrm{p}^{\prime} \mathrm{a}$

where $\S_{0}$ is a constant of the crystal and $a$ is a lattice constant, we obtain

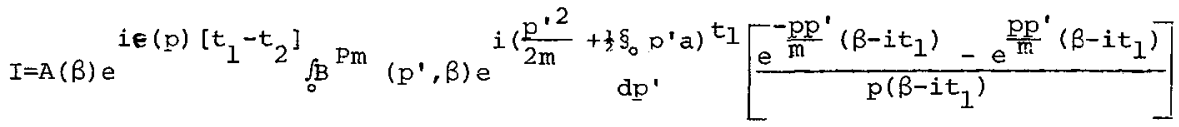

where we have used energy conservation relation $\epsilon(\underline{p}) \pm \S\left(\underline{\underline{p}}^{\prime}\right)=\boldsymbol{\epsilon}\left(\underline{\underline{p}} \pm \underline{\underline{p}}^{\prime}\right)$.

I decays at the most as $\frac{1}{t_{1}}$ or $\frac{1}{t_{2}}$. Thus as $t_{1}, t_{2} \rightarrow \infty$ the whole term vanishes. Thus initial correlations are completely removed from the description of the steady state which is studied only after a long time.

4. The Steady State Transport Equations. Dropping the initial correlations and following the usual procedure ${ }^{l}$, the steady state transport equations can easily be obtained as

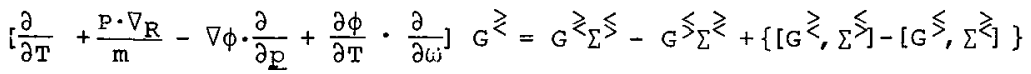

and $\left.\left(\frac{\partial}{\partial T}+\frac{\partial \delta(p)}{\partial p} \cdot \nabla_{R}\right) S^{\gtrless}=\varepsilon^{ \pm} S \$-\varepsilon^{+} S^{\gtrless}+\left[\varepsilon^{ \pm}, S\right\}\right]-\left[\varepsilon^{ \pm}, S^{\gtrless}\right]$

where $\mathrm{G}^{2}$ and $\mathrm{s}^{2}$ stand for electron and phonon steady state Green functions each along with the self energy parts being functions of macroscopic momentum $\underline{p}$, particle energy $\omega$, position $R$ and time $T$. The square brackets are generalized Poisson brackets. These equations are Markoffian and homogeneous. They can be used for the study of various transports. Here we shall use them to study the role of phonon drag in electrical conduction.

5. Phonon Drag. The equations (10) can be linearized in the electric field. To see the effect of the phonon drag, the linear parts of the Green functions $G_{L}^{k}$ and $S_{L}^{\gtrless}$ can be expanded for a homogeneous system as

$G_{L}^{\gtrless}(\underline{p}, \omega)=\sum_{n=0}^{\infty} \lambda^{n} G_{L n}^{\gtrless}(\underline{p}, \omega) ; s_{L}^{\gtrless}(\underline{p}, \omega)=\sum_{n=0}^{\infty} \lambda^{n} S_{L n}^{\gtrless}(\underline{p}, \omega)$

Substitution of these into the linear equations and comparing the terms of the same order of $\lambda$ yields in the lowest order

$$
\begin{aligned}
& G_{L O}^{\gtrless}=\frac{e^{E}}{v} \cdot \frac{\partial}{\partial \underline{p}} G_{E q}^{\gtrless}(\underline{p}, \omega), s_{L 0}^{\gtrless}=0=G_{L I}^{\gtrless}=s_{L I}^{\gtrless} \text {, }
\end{aligned}
$$

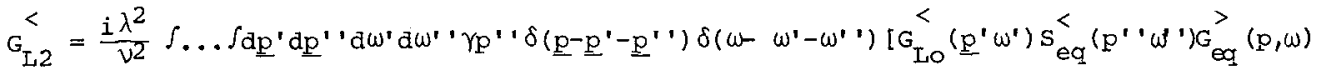

$$
\begin{aligned}
& \left.+ \text { similar terms in } G_{L}^{\gtrless}\right]+\delta\left(\underline{p}^{2} \underline{p}^{\prime}+p^{\prime} \eta \delta\left(\omega-\omega^{\prime}+\omega^{\prime}\right)\left[G_{L o}^{<}\left(p^{\prime} \omega^{\prime}\right) S_{e q}^{>}\left(p^{\prime} \cdot \omega^{\prime}\right) G_{e q}^{>}(p, \omega)\right.\right. \\
& + \text { similar terms in } G_{\mathrm{L}}^{\gtrless}
\end{aligned}
$$

where $\nu$ is the frequency of the field and $G_{\text {eq, }}^{\gtrless}, s_{\text {eq }}^{\gtrless}$ are equilibrium Green functions. It is interesting to see that $G_{L 2}^{<}$has no phonon drag term. It is also a term linear in electron density. Thus in dilute systems like semiconductors, phonon drag has no contribution to the conductivity but the same cannot be said in metals. 
TABLE I. Simplest self-energy diagrams and the corresponding expressions.

\begin{tabular}{|c|c|c|}
\hline Symbol & Simplest Diagram & Expression in the second order \\
\hline$\varepsilon^{<}(1,3)$ & & $\begin{array}{c}\frac{\lambda^{2}}{\Omega} \int d p_{4} \int d p_{5} \gamma_{p_{4}}^{1 / 2} \gamma_{p_{5}}^{1 / 2} g^{<}\left(p_{1}-\underline{p}_{4} t_{1}, p_{3}-p_{5} t_{3}\right) \\
\times n^{<}\left(\underline{p}_{4} t_{1}, \underline{p}_{5} t_{3}\right)\end{array}$ \\
\hline$\varepsilon^{>}(1,3)$ & & $\begin{array}{c}\frac{\lambda^{2}}{\Omega} \int d \underline{p}_{4} \int d \underline{p}_{5} \gamma_{p_{4}}^{1 / 2} \gamma_{p_{5}}^{1_{2}} g^{2}\left(\underline{p}_{1}-\underline{p}_{4} t_{4} \underline{p}_{3}-\underline{p}_{5} t_{3}\right) \\
x n^{>}\left(\underline{p}_{4} t_{1}, \underline{p}_{5} t_{3}\right)\end{array}$ \\
\hline$\varepsilon^{-}(1,3)$ & & $\begin{array}{c}\frac{\lambda^{2}}{\Omega} \int d \underline{p}_{4} \int d \underline{p}_{5} \gamma_{p_{4}}^{1 / 2} \gamma_{p_{5}}^{1 / 2} g^{<}\left(p_{1}-p_{4} t_{1}, p_{3}-p_{5} t_{3}\right) \\
x n^{>}\left(\underline{p}_{4} t_{1}, p_{5} t_{3}\right)\end{array}$ \\
\hline$\varepsilon^{+}(1,3)$ & & $\begin{array}{c}\frac{\lambda^{2}}{\Omega} \int d \underline{p}_{4} \int d \underline{p}_{5} \gamma^{1 / 2} p_{4} \gamma^{1 / 2} p_{5} g^{2}\left(\underline{p}_{1}-\underline{p}_{4} t_{1}, p_{3}-\underline{p}_{5} t_{3}\right) \\
x n^{n}<\left(\underline{p}_{4} t_{1}, \underline{p}_{5} t_{3}\right)\end{array}$ \\
\hline$\varepsilon^{\gg}(1,3)$ & & $\begin{array}{c}\frac{\lambda^{2}}{\Omega} \int d \underline{p}_{4} \int d \underline{p}_{5} \gamma_{p_{4}}^{1 / 2} \gamma_{p_{5}}^{1 / 2} g^{2}\left(\underline{p}_{1}-\underline{p}_{4} t_{1}, \underline{p}_{3}-\underline{p}_{5} t_{3}\right) \\
\times g^{<}\left(\underline{p}_{4} t_{1}, \underline{p}_{5} t_{3}\right)\end{array}$ \\
\hline$\varepsilon^{\ll}(1,3)$ & & $\begin{array}{c}\frac{\lambda^{2}}{\Omega} \int d \underline{p}_{4} \int d \underline{p}_{5} \gamma_{p_{4}}^{1 / 2} \gamma_{p_{5}}^{1 / 2} g^{q}\left(\underline{p}_{1}-\underline{p}_{4} t_{1}, \underline{p}_{3}-\underline{p}_{5} t_{3}\right) \\
\times g_{\left(\underline{p}_{4} t_{1}, \underline{p}_{8} t_{3}\right)}^{>}\end{array}$ \\
\hline
\end{tabular}

\section{References}

1. L. Kadanoff \& G. Baym, The Quantum Statistical Mechanics (Benjamin, N.Y. 1962) .

2. S. Fujita, Phys. Rev. A4, 1114 (1971).

3. C. Bloch \& D. deDomenicis, Nucl. Phys. 7, 459 (1958). 